\section{IDDF2021-ABS-0151 A RETROSPECTIVE ANALYSIS OF PACLITAXEL (ALBUMIN BINDING TYPE) COMBINED WITH S-1 COMPARED WITH SOX REGIMEN AS FIRST-LINE TREATMENT FOR LAUREN DIFFUSE TYPE ADVANCED GASTRIC CANCER}

Yunqi Hua*, Chenlin Wang, Meili Zhao, Kun Cao, Xi Li, Jie Li. Baotou Tumor Hospital, Clinical Oncology of Baotou Medical College, China

\subsection{6/gutjpl-2021-IDDF.160}

Background To investigate the efficacy and safety of Paclitaxel (albumin binding type) combined with S-1 compared with SOX regimen in the treatment of Lauren diffuse-type advanced gastric cancer.

Methods The clinical data of 50 patients with advanced Lauren type diffuse gastric cancer in our hospital from August 2018 to June 2020 were retrospectively analyzed. According to the first-line treatment plan, they were divided into treatment group $(n=25)$ and control group $(n=25)$. The treatment group was treated with Paclitaxel (albumin binding type) combined with S-1 compared chemotherapy. The control group was treated with Oxaliplatin combined with S-1 chemotherapy. Then the adverse reaction and clinical efficacy were recorded. Results Total 50 patients were evaluated for efficacy. The disease control rate of the treatment group was $68.0 \%(17 / 25)$, and the control group was $48.0 \%(12 / 25)$. The disease control rate of the treatment group was higher than the control group, and the difference was statistically significant $(\mathrm{P}<$ $0.05)$. The main side effects of the two groups were myelosuppression and gastrointestinal reactions, mostly grade I-II. The incidence of leucopenia, hemoglobin reduction and thrombocytopenia in the treatment group was significantly lower than that in the control group $(\mathrm{P}<0.05)$. Other toxicities were similar.

Conclusions Paclitaxel (albumin binding type) combined with S-1 compared with SOX regimen has a significant effect in the first-line treatment of advanced Lauren type diffuse gastric cancer, with no increase in the incidence of adverse reactions, has good safety. It is worthy of clinical promotion. Although the efficacy was better, it should be confirmed by well-controlled randomized clinical trials with more patients.

\section{IDDF2021-ABS-0152 GALLBLADDER POLYPS ARE ASSOCIATED WITH GASTROINTESTINAL POLYPS}

Wenbin Geng*, Xiaoyong Wang. The Affiliated Changzhou No. 2 People's Hospital of Nanjing Medical University, China

\subsection{6/gutjnl-2021-IDDF.161}

Background To date, it is not clarified whether patients with gallbladder diseases need gastroscopy and colonoscopy screening. The objective of this study is to retrospective determine the association between gallbladder diseases and gastrointestinal polyps.

Methods A retrospective cross-sectional study included patients who underwent gastroscopy, colonoscopy and abdominal ultrasound as a part of health check-up from January 2015 to July 2020. Gastroscopy and colonoscopy results were divided into four groups by endoscopic findings: polyps-free group, gastric polyps group, colorectal polyps group and gastric plus colorectal polyps group. We compared patients with and without gallbladder diseases for the prevalence of gastrointestinal polyps. The odds ratios (OR) were computed by logistic regression analysis after multivariable adjustment.

Results Totally 1652 patients were included, with 958 patients in the polyps-free group, 504 in the colorectal polyps group, 109 in the gastric polyps group and 91 in the gastric plus colorectal polyps group. Patients with gallbladder polyps had greater odds of having colorectal polyps (adjusted $\mathrm{OR}=1.77$, $95 \%$ confidence interval [Cl]: 1.23 to $2.54, \mathrm{p}=0.002)$ and gastric plus colorectal polyps (adjusted $\mathrm{OR}=2.94,95 \% \mathrm{Cl}$ : 1.62 to $5.32, \mathrm{p}<0.001)$ than those without. Subgroup analysis found that patients with gallbladder polyps larger than $0.5 \mathrm{~cm}$ had greater odds of having colorectal polyps (adjusted

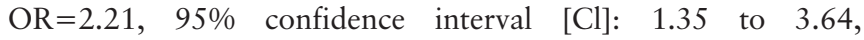
$\mathrm{p}=0.002$ ) and gastric plus colorectal polyps (adjusted $\mathrm{OR}=3.16,95 \%$ confidence interval $[\mathrm{Cl}]: 1.39$ to 7.17 , $\mathrm{p}=0.006)$ and that women with gallbladder polyps had higher prevalence of colorectal polyps $(\mathrm{OR}=2.13,95 \% \mathrm{CI}$ : 1.20 to $3.77, \mathrm{p}=0.010)$ as well as gastric plus colorectal polyps $(\mathrm{OR}=3.69,95 \% \mathrm{CI}: 1.58$ to $8.62, \mathrm{p}=0.003)$. We did not find a positive correlation between gallbladder stones and gastrointestinal polyps.

Conclusions The presence of gallbladder polyps is significantly associated with colorectal polyps and gastric plus colorectal polyps. Gastroscopy and colonoscopy screening might be considered in patients with gallbladder polyps, especially females.

\section{IDDF2021-ABS-0157 ENDOSCOPIC FOREIGN BODYREMOVAL - AN INDIAN EXPERIENCE OVER THREE YEARS}

Deepti Vishwanathan*, Vikas Pandey, Vikramaditya Rawat, Shamsher Singh Chauhan, Rahulkumar Jadhav, Vipul Chaudhari, Swapnil Walke, Chaitanya Atre, Alisha Chaubal, Kiran B, Mit Shah, Chintan Tailor, Harshad Khairnar, Kailash Kolhe, Meghraj Ingle. Lokmanya Tilak Municipal Medical College, Sion, Mumbai, India

\subsection{6/gutjpl-2021-IDDF.162}

Background Endoscopic foreign body (FB) removal forms an important aspect of emergency \& routine endoscopic procedures. This is an analysis of the types of FB encountered \& the experience with their removal over a period of three years.

Methods This is a retrospective analysis of endoscopies performed for FB removal. The data recorded was the age, sex, symptoms, nature of the FB, bowel injury \& endoscopic success/failure.

Results A total of $225 \mathrm{FBs}$ in 225 patients, $65.77 \%$ within the reach of the endoscope were removed from 2017 to 2020, $>95 \%$ being accidental ingestions. Male to Female ratio was 1.25:1. The median age was 6 years (the oldest: 67 years \& the youngest: 4 months old).24.00\% of the total FBs were sharps(GroupS), 67.11\% non-sharps(GroupNS) \& 8.88\% corrosives(GroupC). Almost two thirds (62.66\%) of FBs had a diameter $>2 \mathrm{~cm} \mathrm{\&} 12 \%$ had a length $>5 \mathrm{cms}$. Bowel injury was present in $20.37 \%$ of GroupS $(p=0.030), 4.63 \%$ of GroupNS $(\mathrm{p}<0.05)$ and $45 \%$ of GroupC FB $(\mathrm{p}<0.05)$. The total no of patients with symptoms were $8.88 \%$ with $75 \%$ having dysphagia. $44 \%$ of patients with bowel injury had symptoms and $3.5 \%$ without injury had symptoms $(p<0.05)$. Off the 78 FBs that weren't within the reach of the endoscope, 65.38\% had diameter $>2 \mathrm{~cm}(\mathrm{p}=0.53), 8.97 \%$ had length $>5 \mathrm{~cm} \quad(\mathrm{p}=0.30)$, with $19.23 \% \quad(\mathrm{p}=0.22), \quad 71.79 \%$ $(p=0.27) \& 8.97 \%(p=0.97)$ being from Groups S, NS \& C respectively. We failed in the removal of only 2/147 FBs 